\title{
Management of Influenza Symptoms in Healthy Adults
}

\section{Cost-effectiveness of Rapid Testing and Antiviral Therapy}

Michael B. Rothberg, MD, MPH, Shunian He, MD, David N. Rose, MD

OBJECTIVE: To determine the cost-effectiveness of rapid diagnostic testing and empiric antiviral therapy for healthy adults with symptoms of influenza.

DESIGN: Cost-effectiveness analysis using a decision model based on previously published data. Outcome measures included costs and quality-adjusted life expectancy.

SETTING: Physician's office. adults $<65$ years of age presenting with cough and fever during the influenza season.

INTERVENTIONS: Rapid testing or clinical diagnosis followed by treatment with amantadine, rimantadine, oseltamivir, or zanamivir compared with no antiviral therapy.

RESULTS: Base-case analysis: not giving antiviral therapy is the most expensive and least effective strategy, costing $\mathbf{\$ 4 7 1}$ per patient, mostly owing to time lost from work. Amantadine treatment increases life expectancy by 0.0014 quality-adjusted life years (QALYs) while saving $\$ 108$ per patient relative to no antiviral therapy. Zanamivir is slightly more effective than amantadine, adding 0.0002 QALYs at an incremental cost of $\$ 31$, or $\$ 133,000$ per QALY saved. All other strategies, including testing strategies, are both less effective and more expensive.

SENSITIVITY ANALYSIS: The model is sensitive to the probability of influenza infection, proportion of influenza caused by type $B$, the relative efficacy of the various drugs, and the value of a workday. At a clinical probability of influenza infection $>20 \%$, antiviral therapy is favored. As the proportion of influenza $B$ increases, zanamivir is favored over amantadine. Testing is rarely indicated. Ignoring the costs of lost workdays, amantadine treatment costs $\$ 1,200 /$ QALY saved.

CONCLUSIONS: Antiviral therapy with either amantadine or zanamivir is cost-effective for healthy, young patients with influenza-like illness during the influenza season, depending on the prevalence of influenza $B$.

KEY WORDS: influenza; cost-effectiveness; antiviral therapy; neuraminidase.

J GEN INTERN MED 2003; 18:808-815.
PATIENTS/PARTICIPANTS: Hypothetically healthy, working

$I^{n}$ nfluenza virus infection typically occurs in winter epidemics, causing an estimated 20,000 deaths and more than 100,000 hospitalizations annually in the United States. ${ }^{1,2}$ Although vaccination efforts have been aimed largely at the elderly, the majority of cases and hospitalizations occur among persons younger than 65 years old. ${ }^{2}$ In addition, influenza accounts for $\$ 1$ to $\$ 3$ billion in direct medical costs and $\$ 10$ to $\$ 15$ billion in indirect costs, including lost productivity. ${ }^{3}$ Antiviral drugs for influenza infection have been available for more than 35 years. ${ }^{4}$ Amantadine and rimantadine, which are active only against influenza A infection, have been shown to decrease the duration of illness by approximately 1 day. ${ }^{4}$ A 5-day course of amantadine costs $\$ 2$ and side effects are similar to placebo. ${ }^{4}$ Resistant strains emerge rapidly in treated patients, though the impact of this resistance is unknown. ${ }^{1}$

In 1999, the neuraminidase inhibitors zanamivir and oseltamivir, both active against influenza A and B, were licensed in the United States. Well-conducted studies demonstrate that treatment with either drug reduces the duration of influenza symptoms in average-risk patients by 1 to 1.5 days. ${ }^{5-10}$ In addition, both drugs reduce the incidence of complications requiring antibiotics. ${ }^{7,10}$ Side effects and the emergence of drug resistance are uncommon. ${ }^{11}$ However, these newer agents are expensive, ranging from $\$ 48$ to $\$ 60$ for a 5-day course.

To be effective, antiviral therapy must be started within 48 hours of symptom onset. Unlike traditional viral cultures, which take several days to grow, newer rapid tests can diagnose influenza in the office in less than $30 \mathrm{~min}$, facilitating immediate treatment. Four rapid tests are available, each with a different sensitivity and specificity. All tests can detect both influenza A and B, and one, Directigen AB (BD Diagnostic Systems, Sparks, Md), can differentiate between the two, allowing the physician to reserve treatment with a neuraminidase inhibitor for patients with influenza B infection. ${ }^{12}$ The tests cost between $\$ 15$ and \$25.

Is antiviral therapy cost-effective for healthy patients with influenza-like illness? If so, should treatment be based on clinical diagnosis or directed by rapid testing, and which test should be employed? Should standard therapy include the newer agents, or should they be reserved for patients with proven influenza B infection? In response to these questions, we constructed a decision-analytic model to determine the cost-effectiveness of empiric versus testguided antiviral therapy compared to no antiviral therapy for patients presenting with symptoms of influenza.

\section{METHODS}

Annual Meeting, May 6, 2000, Boston, Mass.

Address correspondence and requests for reprints to Dr. Michael B. Rothberg, MD, MPH, Division of General Medicine and Geriatrics, Baystate Medical Center, 759 Chestnut Street, Springfield, MA 01199 (e-mail: Michael.Rothberg@bhs.org). 


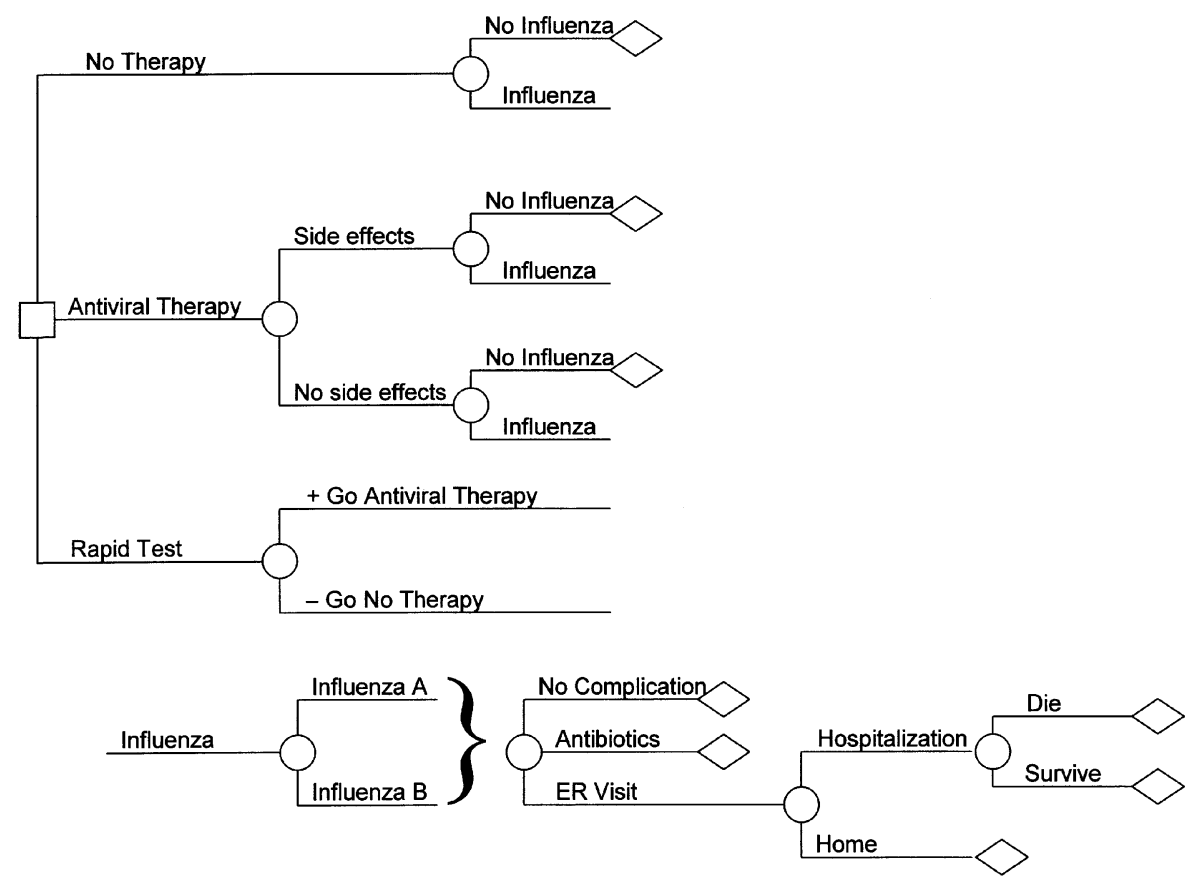

FIGURE 1. Decision model for diagnosis and treatment of influenza-like illness. Square denotes decision node; circles denote chance events; diamonds denote terminal nodes. The actual model contains four rapid test branches and four antiviral therapy branches, representing all four tests and antiviral drugs.

Group, Boston, Mass) to compare the following strategies: (1) no antiviral therapy; (2) empirical treatment with either amantadine, rimantadine, oseltamivir, or zanamivir; (3) rapid testing with one of the nondiscriminating tests followed by treatment with one of the four antiviral drugs; and (4) rapid testing with Directigen $A B$, followed by treatment with amantadine or rimantadine for influenza A infection and zanamivir or oseltamivir for influenza B infection. A graphical representation of the model is shown in Figure 1. We assumed that all drugs would be initiated within 48 hours of symptom onset and continued for 5 days at doses recommended by the manufacturers.

For the reference case, we considered unvaccinated, healthy, working adults between 20 and 50 years of age presenting with influenza-like illness during the influenza season. The model considers the prevalence of influenza, sensitivity and specificity of the tests, and the following adverse events: antiviral side effects, influenza complications requiring antibiotics, emergency room visits, hospitalizations, and deaths. Influenza infection is divided into types A and B, which we assumed to be of equal severity. We assumed that only neuraminidase inhibitors would be effective in treating influenza B. Outcomes were expressed in dollars per quality-adjusted life year (BALY) saved.

\section{Data and Assumptions}

Baseline estimates and range for sensitivity analyses are provided in Table 1.
Influenza Prevalence and Complications. Influenza occurs in winter epidemics. For our base case, we analyzed patients presenting during the peak flu season (usually December through March in the northern hemisphere) in a region with documented influenza cases. In such a setting, patients with influenza-like illness, defined as abrupt onset of fever $>37.8^{\circ} \mathrm{C}$ plus 2 of 4 symptoms (cough, myalgia, sore throat, and headache) have a $70 \%$ to $87 \%$ chance of having influenza. ${ }^{13-15}$ If influenza has not been documented in the area, the positive predictive value of these symptoms drops to between $44 \%$ and $60 \% .^{7,16}$ During the periinfluenza season (usually October, November, April, and May), patients with the same symptoms have less than a $20 \%$ chance of having influenza. ${ }^{17}$

The proportion of influenza infections caused by influenza B changes every year. In 2002, approximately $11 \%$ of influenza specimens were type B. In past years, the proportion has been as high as $63 \%$ in some regions. ${ }^{17} \mathrm{We}$ used 2001-02 as our base season, but tested a broad range of proportions in the sensitivity analysis.

Complications requiring antibiotics were based on the experience of subjects in the placebo arms of treatment trials. ${ }^{7,18}$ We used published estimates for hospitalization rates of healthy young women ${ }^{19}$ and healthy patients under 65 years of age. ${ }^{2,20}$ These correlate closely with the hospitalization rate among placebo recipients in the zanamivir treatment trials. ${ }^{21}$ The death rate was expressed as a function of the hospitalization rate, as described by Simonsen et al. ${ }^{2}$ 
Table 1. Baseline Values for the Decision Model and Ranges Used in Sensitivity Analysis

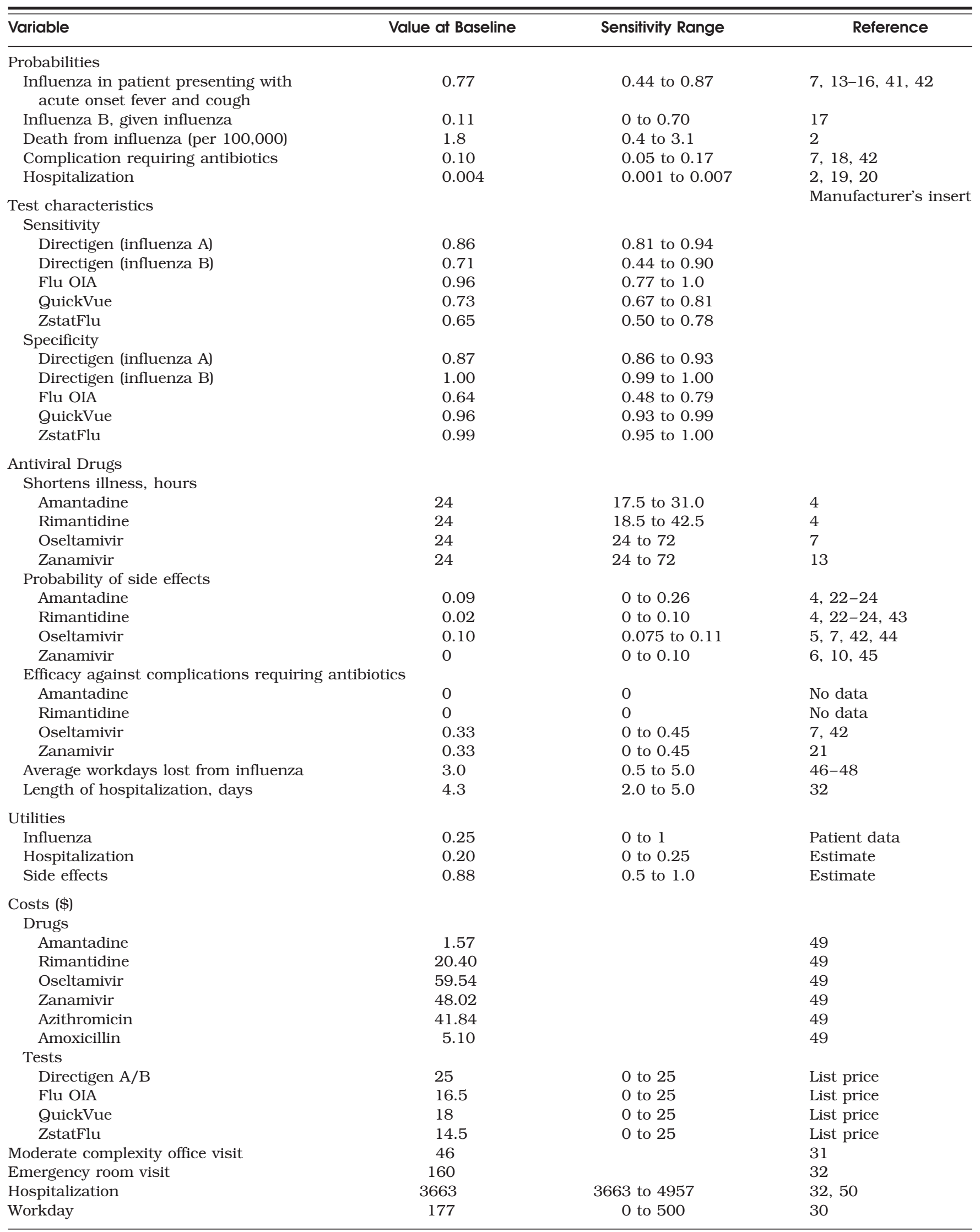


Diagnostic Tests. Our estimates of the rapid tests' characteristics were based on information provided by the manufacturers. Although the best results are obtained by nasopharyngeal wash or aspiration, for the base case we assumed that physicians would use nasal or nasopharyngeal swabs.

Efficacy of Treatment. The benefit of anti-influenza drugs was taken from randomized, placebo-controlled trials of average-risk subjects with naturally occurring infection. Although the study designs and durations of illness in the placebo groups varied among trials, each drug was found to reduce the duration of illness among infected subjects by 1 to 1.5 days. ${ }^{4,7,13}$ Other than two small studies of amantadine and rimantadine, ${ }^{22,23}$ there are no comparative trials. Given the lack of uniformity in study design and outcome measurement, it is not possible to say that one drug is more effective than another in shortening the illness. The effect of the drugs on antibiotic use for bacterial complications was documented only in the neuraminidase inhibitor studies. Zanamivir significantly reduced antibiotic use from $17 \%$ in the placebo group to $11 \%$ in the zanamivir group. ${ }^{21}$ Oseltamivir had a similar effect. ${ }^{7}$ No study was sufficiently powered to detect a difference in hospitalizations or mortality. Therefore, in the base case, we assumed that no drug would prevent hospitalization or death. We tested this assumption in the sensitivity analysis.

Adverse Effects. Rates of adverse effects of the antiviral drugs were taken from the clinical trials of efficacy. Thirteen percent of healthy adults taking amantadine and 6\% of those taking rimantadine experienced CNS side effects compared with $4 \%$ of those taking placebo. ${ }^{24}$ Oseltamivir was associated with nausea and vomiting (13\% in the oseltamivir vs $3 \%$ in the placebo group). ${ }^{7}$ Zanamivir was not associated with adverse events during clinical trials. We estimated that adverse effects would last for 2 days.

Utilities. Utilities are used to represent the value of a health state, to allow comparison of different health states and in calculation of quality-adjusted life expectancy. Utilities are typically expressed on a scale of 0 to 1 , with 0 representing death and 1 representing full health. Estimates for the utility of influenza illness in adults have ranged from $-0.06^{25}$ using the Eurobol method to $0.6^{26,27}$ using the Quality of Well-Being Index. In our adult general medical clinic, we administered the health utilities index (HUI-3) ${ }^{28}$ to 15 randomly selected working-age patients and health care workers with a history of influenza. We asked them to rate their health state during their most recent influenza-like illness, based on their recollection. Their mean response constituted our base case utility for a day of influenza illness. Utilities for hospitalization and drug side effects were also derived from the HUI-3.

Costs. We used a societal perspective in keeping with the recommendations of the Panel on Cost-Effectiveness in
Health and Medicine. ${ }^{29}$ Thus, we considered direct medical costs, including physician visits, diagnostic tests, medications, and hospitalizations, as well as indirect costs in the form of lost productivity as a result of illness, using the average hourly cost of compensation for all civilian workers. ${ }^{30}$ Physician fees were based on a moderate-complexity office visit for an established patient. ${ }^{31}$ We used the retail price provided from the manufacturer for the diagnostic tests. Medication costs were average wholesale prices, plus 30 minutes of an average worker's time to fill the prescription. Hospitalization costs were based on 333 patients hospitalized for influenza at 75 different hospitals. ${ }^{32}$ All costs are updated to 2001 dollars using the medical care component of the Consumer Price Index. ${ }^{33}$

\section{RESULTS}

\section{Base Case Analysis}

Although we tested all four drugs in the sensitivity analysis, in the base case we present only strategies that include amantadine or zanamivir, because rimantadine is more expensive than amantadine and oseltamivir is more expensive than zanamivir, and there is no evidence that any drug is more effective than the others. Not giving antiviral therapy is the most expensive and least effective of all the strategies, costing \$471 per patient. The high cost is comprised mostly of time lost from work, and is consistent with the huge economic burden engendered by annual influenza epidemics. Treating all patients with amantadine increases life expectancy by 0.0014 QALYs and saves $\$ 108$ per patient relative to no antiviral therapy. It is also the least expensive of all the strategies. Relative to treatment with amantadine, treating all patients with zanamivir saves an additional 0.0002 QALYs at a marginal cost of $\$ 31$, or $\$ 133,000$ per BALY saved. Figure 2 shows the costs and benefits of all 11 strategies. The line represents the efficiency frontier, which connects the only two cost-effective strategies using base case assumptions: no test, treat all with amantadine, and no test, treat all with zanamivir. The slope of the line is the marginal cost-effectiveness ratio of treating all patients with zanamivir compared to amantadine. All testing strategies, as well as the strategy of no antiviral therapy, lie to the right and below the frontier, indicating that they increase costs and decrease health.

\section{Sensitivity Analysis}

Probability of Influenza Infection and Prevalence of Influenza Type B. Figure 3 depicts the optimal strategy based on a societal willingness to pay $\$ 50,000$ per QALY saved. When the probability of influenza infection is high $(>40 \%)$, as during peak influenza season, the preferred treatment depends on the proportion of cases caused by influenza B. When the proportion is low (as in 2002, the base case), treating all patients with amantadine (the white area) is less expensive and more effective than no antiviral therapy. Zanamivir is slightly more effective than amantadine, but 


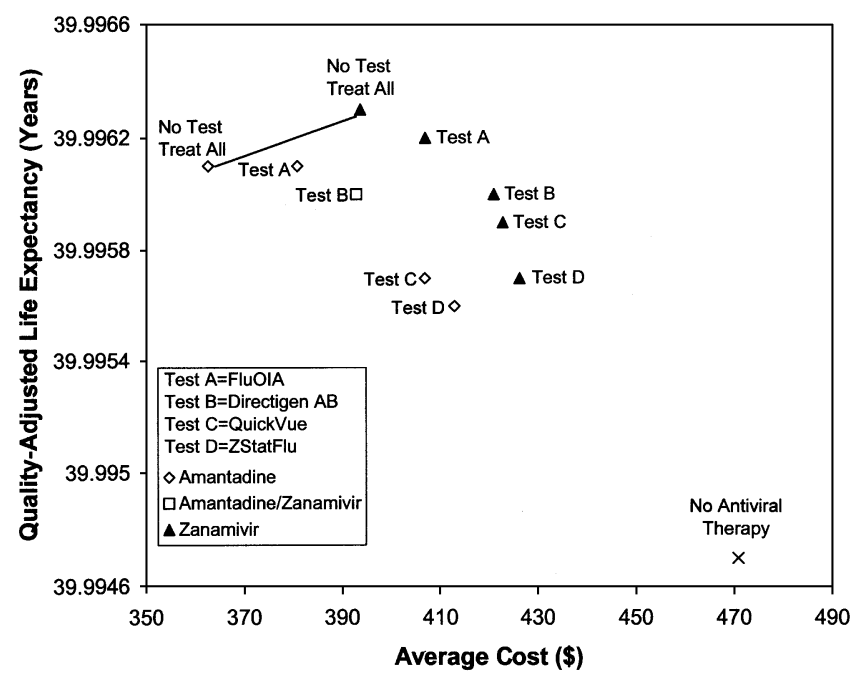

FIGURE 2. Incremental cost-effectiveness of 11 testing and treatment strategies for a 38-year-old patient with influenza-like illness. The testing strategies begin by testing all patients; those with positive results are treated with the drug shown.

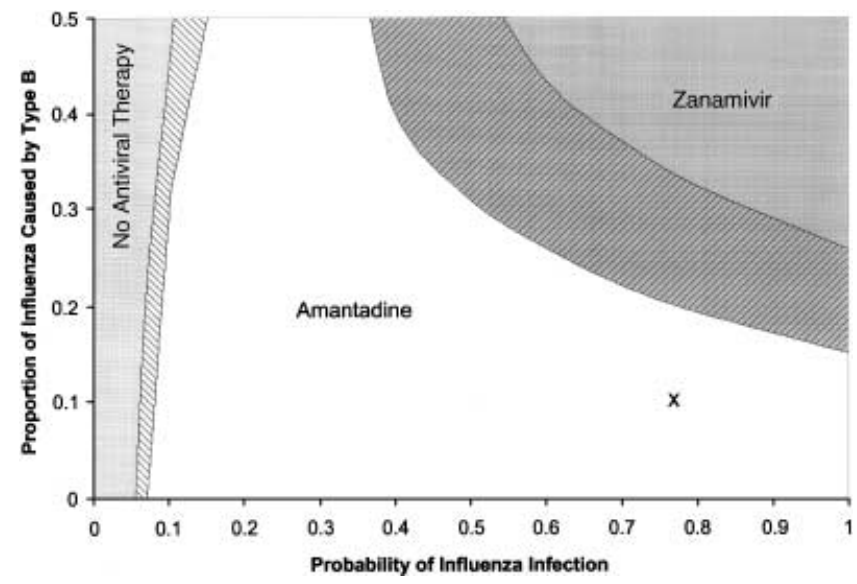

FIGURE 3. Cost-effectiveness of antiviral therapy as a function of the probability of influenza infection and the proportion of influenza caused by type B. Solid areas show strategies that are cost-saving. Hatched areas have marginal cost-effective ratios between $\$ 0$ and $\$ 50,000$ per quality-adjusted life years saved.

prohibitively expensive (usually $>\$ 100,000 /$ QALY saved). As influenza B accounts for a higher proportion of influenza cases, treating all patients with zanamivir becomes increasingly cost-effective (dark gray hatched area) and finally cost-saving (dark gray area). When the probability of influenza infection is low ( $10 \%$ to $40 \%$ ), as in the peri-influenza season, treatment with amantadine is cost-saving even if influenza B accounts for $40 \%$ of influenza cases.

Efficacy of Treatment. The model is sensitive to differences in the relative efficacy of the antiviral drugs. If one drug were to shorten the course of the illness by even 5 hours more than the others, under most circumstances it would offer the best outcome at the lowest cost. On the other hand, assuming that neuraminidase inhibitors prevent complications or hospitalization had minimal effect, because compared to missing work these are rare events.

Medication Side Effects. In treatment studies, side effects of the medications were generally mild, often no worse than placebo. Varying the probability of side effects within the $95 \%$ confidence intervals did not change the results substantially.

Value of a Workday. The model is sensitive to the value of a workday. If a patient's time has no monetary value, then not giving antiviral therapy is the least expensive strategy. Treating all patients with amantadine increases qualityadjusted life expectancy at a cost of \$1200/QALY saved, while treating all patients with zanamivir increases qualityadjusted life expectancy at a marginal cost of $\$ 198,000 /$ BALY saved, relative to treatment with amantadine. If a workday is worth even $\$ 3$, then amantadine is cost-saving relative to no antiviral therapy during the peak influenza season (Fig. 4A, white area). The cost-effectiveness of zanamivir also improves as the value of a workday increases, but even at a value of $\$ 400$ per workday, treating all patients with zanamivir exceeds \$50,000/QALY saved. Again, as the proportion of influenza caused by influenza type B increases, zanamivir becomes increasingly costeffective (hatched gray area) and then cost-saving (dark gray area). During the peri-influenza season (Fig. 4B), amantadine is cost-saving as long as the cost of a work day exceeds $\$ 14$, and empiric zanamivir is never preferred, even when influenza type B is predominant. In that case, rapid testing followed by zanamivir therapy is the preferred strategy.

Utility of Influenza. We varied the utility of influenza illness through the entire range from 0 to 1 . Because quality adjustment does not affect costs, regardless of the utility of influenza, amantadine was always cost-saving compared with no antiviral therapy. Decreasing the utility of influenza illness did slightly improve the cost-effectiveness of the neuraminidase inhibitors. However, even if influenza is assigned a utility of 0 (equivalent to being dead), zanamivir is still expensive, unless influenza B is highly prevalent.

Rapid Testing. Rapid testing for influenza is rarely indicated because the test itself is usually more expensive than the treatment. Moreover, test sensitivity ranges from $65 \%$ to $96 \%$, resulting in a large number of false-negative tests, especially when influenza is prevalent. False-negatives are expensive because they incur the cost of testing but forfeit the economic savings associated with treatment. We found a limited role for testing when the probability of influenza is low, as in the peri-influenza season, and the proportion of influenza B circulating is greater than 50\% (Fig. 4). In that scenario, amantadine is relatively ineffective. Identifying the few patients who will benefit from zanamivir is less expensive than treating all patients with zanamivir. 

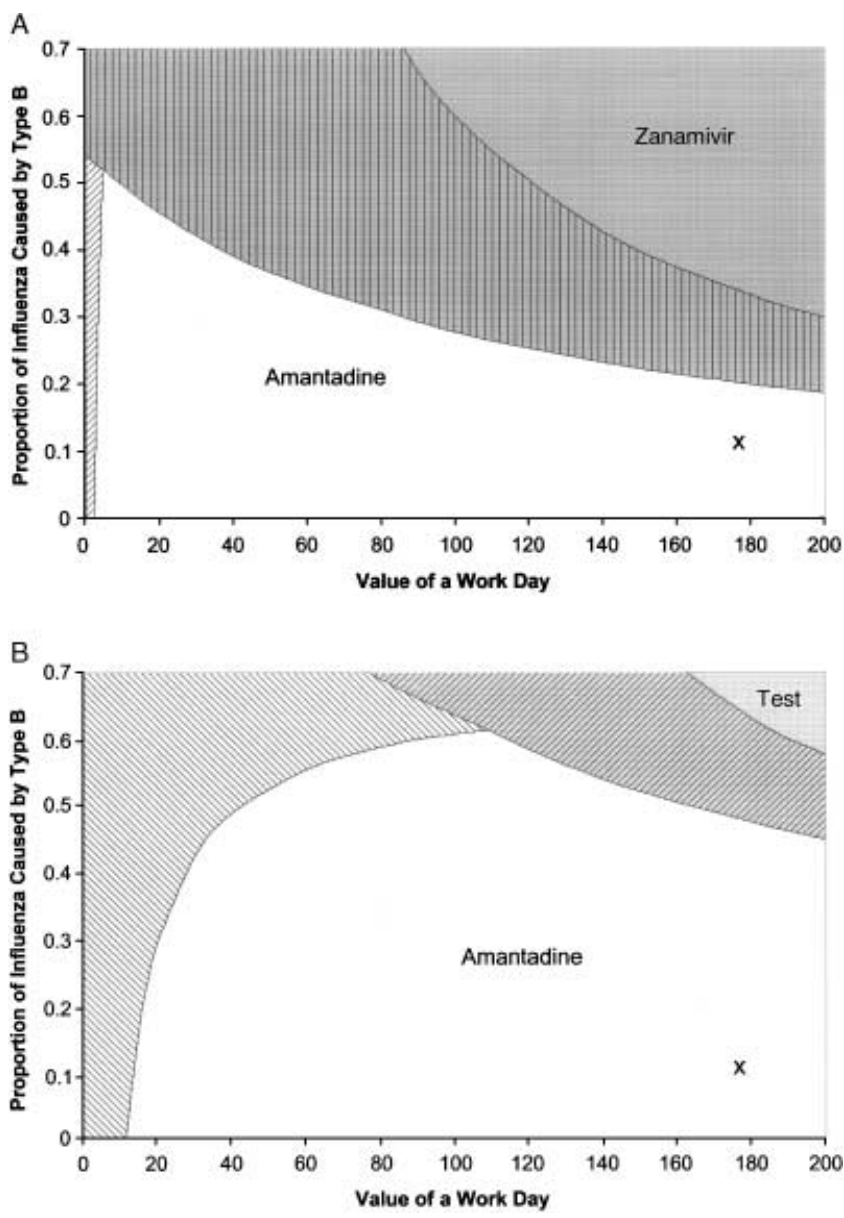

FIGURE 4. A. Cost-effectiveness of antiviral therapy as a function of the value of a workday and the proportion of influenza caused by type B. Solid areas show strategies that are costsaving. Hatched areas have marginal cost-effective ratios between $\$ 0$ and $\$ 50,000$ per quality-adjusted life year saved. The figure assumes probabilities of influenza infection of $77 \%$ and $20 \%$, respectively.

The lowest priced test is usually preferred. If all tests cost the same, then Directigen $A B$ is least expensive, because it allows differential treatment for influenza $\mathrm{A}$ and B. However, this benefit only justifies a $\$ 5$ to $\$ 10$ price premium. On the other hand, FluOIA (Thermo BioStar, Boulder, $\mathrm{CO}$ ) is never preferred at any price because its low specificity results in too many false-positives. Collecting nasopharyngeal specimens by wash rather than swab would not change these results substantially.

\section{DISCUSSION}

Influenza is estimated to strike between $5 \%$ and $10 \%$ of adults annually, resulting in billions of dollars in direct and indirect costs. Although antiviral drugs have been available for decades and are indicated for the treatment of uncomplicated influenza, ${ }^{1}$ these drugs are not widely prescribed. Our decision model, based on data taken from efficacy studies in thousands of patients, demonstrates that the economic impact alone validates the use of antiviral therapy in healthy adults with influenza-like illness. The small benefit of shortening symptoms by an average of 1 day is by no means trivial. If 14 million American workers all return to work 1 day sooner as a result of antiviral therapy, in addition to averting 14 million symptom-days, the annual savings, based on our model, would be $\$ 1.5$ billion. Even for patients who do not work, treating influenza with amantadine costs only $\$ 1200 /$ QALY saved. By comparison, combination therapy for hepatitis C costs $\$ 7000$ per QALY saved, ${ }^{34}$ while screening mammography for women aged 69 years or more costs $\$ 118,000 /$ QALY saved. ${ }^{35}$ Previous influenza models, ${ }^{25,27,36-40}$ which either did not include amantadine or neglected work-related costs, all found antiviral treatment to be cost-effective, but not cost-saving compared to no antiviral therapy, and conclude that its use is justified. Although specific thresholds are offered for probability of influenza infection and prevalence of influenza B, none of these models explores the crucial interaction of these two variables, leaving clinicians to guess as to which drug would be appropriate for a particular patient.

Like other authors, we found that the incremental benefit of using antiviral therapy is small, on the order of 0.0014 BALYs (or 12.2 quality-adjusted hours) gained. Although this benefit may seem small, it is remarkably stable. Only the probability of influenza infection, the proportion of influenza caused by type $\mathrm{B}$, the value of a workday, and the relative efficacy of the drugs have any major impact on the choice of strategy. For example, doubling the incidence of side effects from amantadine would decrease the benefit to 0.0013 QALYs or 11.4 quality-adjusted hours. More importantly, this small benefit does not come at a cost, but actually saves money.

Based on the findings of our study, we offer an algorithmic approach to the management of healthy adults who present with influenza symptoms. The algorithm chooses the most effective strategy with a cost-effectiveness ratio of $\$ 50,000 /$ QALY or less (Fig. 4). During the influenza season, such patients have a high probability of influenza infection, and amantadine treatment is cost-saving. In regions where influenza B exceeds $40 \%$ of influenza cases (or 20\% during a regional epidemic), treatment with a neuraminidase inhibitor offers additional benefit and can be considered cost-effective for working adults (Fig. 5). If more than half of influenza cases are caused by influenza B, then zanamivir is also cost-effective for nonworking adults. In the peri-influenza season, amantadine would still be cost-saving, unless the majority of cases are caused by influenza $\mathrm{B}$, in which case rapid testing followed by treatment with zanamivir is preferred. The proportion of influenza B is reported weekly on the Centers for Disease Control and Prevention's website. ${ }^{17}$

In years or regions when there is little influenza, or if a patient has received influenza vaccine, the probability of influenza infection will be lower than that used in the 


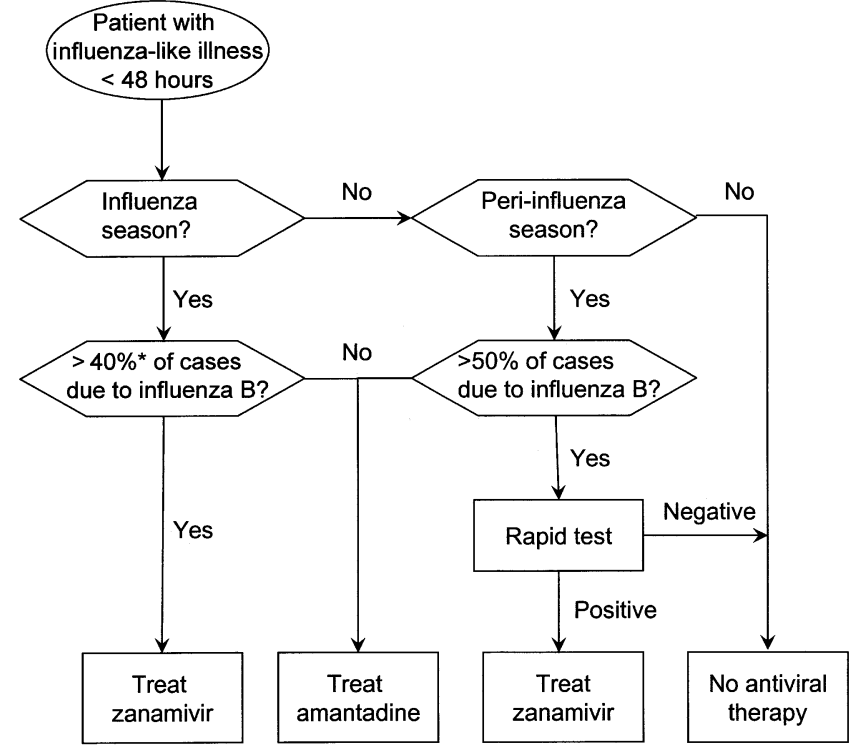

* $>20 \%$ during a regional epidemic

FIGURE 5. Algorithmic approach to the patient with influenzalike illness. All patients begin in the oval at the top of the diagram. Hexagons are influenza conditions that can be answered either "yes" or "no." Rectangles represent tests or treatments. ${ }^{*}>20 \%$ during a regional epidemic.

algorithm. In that case, the preferred treatment can be found by plotting the clinician's estimate of the probability of influenza infection against the proportion of influenza caused by type B on the graph in Figure 3.

Our study has limitations. First, for want of comparative data, we assumed that all drugs have equal efficacy. However, if any drug were even $20 \%$ more effective than the others in reducing the duration of illness, it would usually be the drug of choice. We also assumed that shortening the duration of fever by 1 day results in patients returning to work 1 day sooner. While several studies found that treatment with neuraminidase inhibitors returned patients to normal activity 1-2 days sooner than did placebo, ${ }^{7,13}$ no study reported return to work. Because cost-effectiveness of the neuraminidase inhibitors depends on the economic impact of lost work time, future influenza studies should report the effect of the drugs on subjects' return to work. Amantadine, however, is cost-effective even for nonworking adults.

Other factors, such as the incidence of side effects or whether these drugs prevent influenza complications, did not affect the decision for healthy adults, unless the side effects were so severe that they delayed return to work. Further studies are needed to assess the impact of these drugs on complications in the elderly and the very young, who are disproportionately affected by influenza morbidity and mortality. Indeed, preliminary studies of zanamivir imply that it may have its greatest efficacy in high-risk groups. ${ }^{10}$

Our analysis did not address vaccinated patients, as there is currently no recommendation for or against vaccination in healthy persons less than 50 years of age. In years when the vaccine is well-matched to circulating strains, vaccinated patients presenting with influenza-like illness should have a significantly lower probability of influenza than unvaccinated patients, making antiviral therapies less cost-effective. However, by estimating the probability of influenza in any given patient, vaccinated or not, the preferred therapy can be chosen based on Figure 3.

Studies have shown that resistance to amantadine and rimantadine may develop rapidly. ${ }^{1}$ While there is no evidence that this would lead to resistant strains in subsequent years, effectiveness of these medications may decrease as the influenza season progresses. If so, the neuraminidase inhibitors might become more useful, as they seem less likely to induce resistance. ${ }^{1}$

All of the cost-effective strategies involve treatment based on clinical diagnosis. We did find a limited role for testing when the probability of influenza infection is low, as in the peri-influenza season, and a majority of cases are caused by influenza B. In this setting, tests with high specificity are most cost-effective. Directigen $A B$ offers the additional benefit of distinguishing between influenza A and $\mathrm{B}$ and would be preferred over other tests if the difference in the cost of testing were $\$ 5$ or less.

Treatment based on clinical diagnosis will surely result in many patients without influenza taking antiviral drugs. Depending on the season and the region, the numberneeded-to-treat to shorten the illness by 1 day ranges from 1.25 to 10.0. Anti-influenza drugs have a safety profile similar to or better than antibiotics and nonsteroidal antiinflammatory agents, which are routinely prescribed for patients with influenza-like illness. Unlike antibiotics, antiinfluenza drugs should not induce resistance in patients who do not have influenza. Furthermore, patients offered antivirals would not only benefit from effective treatment, they might be less inclined to request antibiotics.

\section{REFERENCES}

1. Bridges CB, Fukuda K, Uyeki TM, Cox NJ, Singleton JA. Prevention and control of influenza. Recommendations of the Advisory Committee on Immunization Practices (ACIP). MMWR Recomm Report 2002;51 (RR-3):1-31.

2. Simonsen L, Fukuda K, Schonberger LB, Cox NJ. The impact of influenza epidemics on hospitalizations. J Infect Dis. 2000;181:8317.

3. Szucs T. The socio-economic burden of influenza. J Antimicrob Chemother. 1999;44 (Suppl. B):11-5

4. Jefferson T, Demicheli V, Rivetti D, Deeks J. Cochrane reviews and systematic reviews of economic evaluations. Amantadine and rimantadine in the prevention and treatment of influenza. Pharmacoeconomics. 1999;16 (Suppl. 1):85-9.

5. Hayden FG, Atmar RL, Schilling M, et al. Use of the selective oral neuraminidase inhibitor oseltamivir to prevent influenza. N Engl J Med. 1999;341:1336-43.

6. Hayden FG, Osterhaus AD, Treanor JJ, et al. Efficacy and safety of the neuraminidase inhibitor zanamivir in the treatment of influenzavirus infections. Gg167 Influenza Study Group. N Engl J Med 1997;337:874-80. 
7. Treanor JJ, Hayden FG, Vrooman PS, et al. Efficacy and safety of the oral neuraminidase inhibitor oseltamivir in treating acute influenza: a randomized controlled trial. US Oral Neuraminidase Study Group. JAMA. 2000;283:1016-24.

8. Monto AS, Fleming DM, Henry D, et al. Efficacy and safety of the neuraminidase inhibitor zanamivirin the treatment of influenza A and B virus infections. J Infect Dis. 1999;180:254-61.

9. Matsumoto K, Ogawa N, Nerome K, et al. Safety and efficacy of the neuraminidase inhibitor zanamivir in treating influenza virus infection in adults: results from Japan. Gg167 Group. Antivir Ther. 1999;4:61-8.

10. The MIST (Manage Influenza Southern Hemisphere Trialists) Study Group. Randomised trial of efficacy and safety of inhaled zanamivir in treatment of influenza A and B virus infections. Lancet. 1998;352:1877-81

11. Anonymous. Two neuraminidase inhibitors for treatment of influenza. Med Lett Drugs Ther. 1999;41:91-3.

12. Johnson JR. Antiviral agents for influenza. JAMA. 2000;283:306970. Letter; Comment

13. Monto AS, Webster A, Keene O. Randomized, placebo-controlled studies of inhaled zanamivir in the treatment of influenza A and B: pooled efficacy analysis. J Antimicrob Chemother. 1999;44 (Suppl. B):23-9.

14. Zambon M, Hays J, Webster A, Newman R, Keene O. Diagnosis of influenza in the community: relationship of clinical diagnosis to confirmed virological, serologic, or molecular detection of influenza. Arch Intern Med. 2001;161:2116-22.

15. Boivin G, Hardy I, Tellier G, Maziade J. Predicting influenza infections during epidemics with use of a clinical case definition. Clin Infect Dis. 2000;31:1166-9.

16. Govaert TM, Dinant GJ, Aretz K, Knottnerus JA. The predictive value of influenza symptomatology in elderly people. Fam Pract. 1998; 15:16-22.

17. Centers for Disease Control and Prevention. Reports \& Surveillance Methods in the United States: Current U.S. Flu Report. Available at http://www.cdc.gov/ncidod/diseases/flu/weeklychoice.htm. Accessed March 26, 2002.

18. Kaiser L, Henry D, Flack NP, Keene O, Hayden FG. Short-term treatment with zanamivir to prevent influenza: results of a placebocontrolled study. Clin Infect Dis. 2000;30:587-9.

19. Neuzil KM, Reed GW, Mitchel EF, Jr, Griffin MR. Influenzaassociated morbidity and mortality in young and middle-aged women. JAMA. 1999;281:901-7.

20. Barker WH, Mullooly JP. Impact of epidemic type A influenza in a defined adult population. Am J Epidemiol. 1980;112:798-811.

21. Kaiser L, Keene ON, Hammond JM, Elliott M, Hayden FG. Impact of zanamivir on antibiotic use for respiratory events following acute influenza in adolescents and adults. Arch Intern Med. 2000; 160:3234-40.

22. Van Voris LP, Betts RF, Hayden FG, Christmas WA, Douglas RG Jr. Successful treatment of naturally occurring influenza A/USSR/ 77, H1N1. JAMA. 1981;245:1128-31.

23. Wingfield WL, Pollack D, Grunert RR. Therapeutic efficacy of amantadine $\mathrm{HCl}$ and rimantadine $\mathrm{HCl}$ in naturally occurring influenza A2 respiratory illness in man. N Engl J Med. 1969;281:579-84.

24. Dolin R, Reichman RC, Madore HP, Maynard R, Linton PN, WebberJones J. A controlled trial of amantadine and rimantadine in the prophylaxis of influenza A infection. N Engl J Med. 1982;307:5804

25. Griffin AD, Perry AS, Fleming DM. Cost-effectiveness analysis of inhaled zanamivir in the treatment of influenza A and B in highrisk patients. Pharmacoeconomics. 2001;19:293-301.

26. Cost-effectiveness of flu vaccine. Congressional Office Technol Assessment. Conn Med. 1982;46:607.

27. Muennig PA, Khan K. Cost-effectiveness of vaccination versus treatment of influenza in healthy adolescents and adults. Clin Infect Dis. 2001;33:1879-85.
28. Feeny D, Furlong W, Torrance GW, et al. Multiattribute and singleattribute utility functions for the health utilities index mark 3 system. Med Care. 2002;40:113-28.

29. Gold M, Siegel J, Russell L, Weinstein M, eds. Cost-Effectiveness in Health and Medicine. New York, NY: Oxford University Press; 1996.

30. U.S. Bureau of Labor Statistics. Employer costs for employee compensation-March 2001. Available at http://www.bls.gov/ news.release/ecec.nrO.htm. Accessed March 26, 2002.

31. Crane M. What you charge vs what you get. Med Econ. 2001;78:55-7.

32. Cox FM, Cobb MM, Chua WQ, Mclaughlin TP, Okamoto LJ. Cost of treating influenza in emergency department and hospital settings. Am J Manag Care. 2000;6:205-14.

33. U.S. Bureau of Labor Statistics. Consumer Price Index-All Urban Consumers. Available at http://www.bls.gov/data/. Accessed May 6, 2002 .

34. Wong JB, Koff RS. Watchful waiting with periodic liver biopsy versus immediate empirical therapy for histologically mild chronic hepatitis C. A cost-effectiveness analysis. Ann Intern Med. 2000;133:665-75.

35. Kerlikowske K, Salzmann P, Phillips KA, Cauley JA, Cummings SR. Continuing screening mammography in women aged 70-79 years: impact on life expectancy and cost-effectiveness. JAMA. 1999;282:2156-63.

36. Mauskopf JA, Cates SC, Griffin AD, Neighbors DM, Lamb SC, Rutherford C. Cost effectiveness of zanamivir for the treatment of influenza in a high risk population in Australia. Pharmacoeconomics. 2000; 17:611-20.

37. Armstrong E, Khan Z, Perry A, Hons M, Perri L. The cost effectiveness of zanamivir and oseltamivir for influenza treatment. Formulary. 2000;35:979-89.

38. Lee PY, Matchar DB, Clements DA, Huber J, Hamilton JD, Peterson ED Economic analysis of influenza vaccination and antiviral treatment for healthy working adults. Ann Intern Med. 2002;137:225-31.

39. Smith KJ, Roberts MS. Cost-effectiveness of newer treatment strategies for influenza. Am J Med. 2002;113:300-7.

40. Sintchenko V, Gilbert GL, Coiera E, Dwyer D. Treat or test first? Decision analysis of empirical antiviral treatment of influenza virus infection versus treatment based on rapid test results. J Clin Virol. 2002;25:15-21.

41. Monto AS. Preventing influenza in healthy adults: the evolving story. JAMA. 2000;284:1699-701.

42. Nicholson KG, Aoki FY, Osterhaus AD, et al. Efficacy and safety of oseltamivir in treatment of acute influenza: a randomised controlled trial. Neuraminidase inhibitor flu treatment investment group. Lancet. 2000;355:1845-50.

43. Hayden FG, Monto AS. Oral rimantadine hydrochloride therapy of influenza A virus H3N2 subtype infection in adults. Antimicrob Agents Chemother. 1986;29:339-41

44. Hayden FG, Treanor JJ, Fritz RS, et al. Use of the oral neuraminidase inhibitor oseltamivir in experimental human influenza: randomized controlled trials for prevention and treatment. JAMA. 1999;282:1240-6.

45. Hayden FG, Treanor JJ, Betts RF, Lobo M, Esinhart JD, Hussey EK. Safety and efficacy of the neuraminidase inhibitor GG167 in experimental human influenza. JAMA. 1996;275:295-9.

46. Kavet J. A perspective on the significance of pandemic influenza. Am J Public Health. 1977;67:1063-70.

47. Keech M, Scott AJ, Ryan PJ. The impact of influenza and influenzalike illness on productivity and healthcare resource utilization in a working population. Occup Med (Lond). 1998;48:85-90.

48. Kumpulainen V, Makela M. Influenza vaccination among healthy employees: a cost-benefit analysis. Scand $J$ Infect Dis. 1997;29:181-5.

49. 2001 Drug Topics Red Book. Montvale, NJ: Medical Economics, Inc; 2001.

50. The Medstat Group. 2000 DRG Guide. Descriptions and Normative Values 2001 . 